\title{
Agreement between People with Aphasia and Their Proxies on Health-Related Quality of Life after Stroke, Using the Greek SAQOL-39g
}

\author{
Maria Ignatiou, Vasiliki Christaki, Evripidis Nicolaos Chelas, \\ Evangelia Antonia Efstratiadou, Katerina Hilari \\ Division of Language and Communication Science, City University London, London, UK \\ Email:k.hilari@city.ac.uk
}

Received June $1^{\text {st }}, 2012$; revised July $2^{\text {nd }}, 2012$; accepted August $3^{\text {rd }}, 2012$

\begin{abstract}
Health related quality of life (HRQL) measures are increasingly used to evaluate stroke interventions. People with severe aphasia after stroke may be unable to self-report on such measures, necessitating the use of proxy respondents. This study explored the level of agreement between people with aphasia and their proxies on the Greek Stroke and Aphasia Quality of Life Scale-39 generic version (SAQOL-39g) and whether this agreement was influenced by proxy levels of depression and carer strain. Methods: Participants were people with aphasia (PWA) who were over six months post-stroke and medically stable. Proxies were nominated by the PWA and had to see them at least twice a week. PWA completed the Frenchay Aphasia Screening Test and the Greek SAQOL-39g. Proxies completed the Greek SAQOL-39g proxy version, the General Health Questionnaire-12 and the Caregiver Strain Index. Results: 23 pairs of people with aphasia and their proxies took part. Proxies rated people with aphasia as more severely affected than they rated themselves. The difference was significant for the overall scale and the physical and communication domains $(p<0.05)$; yet the bias introduced by these differences was small to moderate, with effect sizes ranging from 0.15 to 0.47 . The strength of the agreement between people with aphasia and proxies was excellent for the overall scale and all three domains $(\mathrm{ICC}=0.79-0.97)$. The level of agreement was not associated with carer strain or emotional distress. We conclude that clinicians and researchers can use proxy ratings to evaluate the quality of life of people with severe aphasia but need to be aware of trends in proxy reporting and take these into account when interpreting data.
\end{abstract}

Keywords: Proxy; Stroke Outcome; Health-Related Quality of Life; Aphasia; SAQOL-39g

\section{Introduction}

Health-related quality of life (HRQL) is defined as the impact of a health state on a person's ability to lead a fulfilling life (Bullinger, Anderson, Cella, \& Aaronson, 1993) and generally incorporates the individual's subjective evaluation of their physical, mental/emotional, family and social functioning (Berzon, Hays \& Shumaker, 1993; Hays, Anderson, \& Revicki, 1993). HRQL measures are increasingly used to evaluate healthcare interventions and they can be particularly useful for people with chronic conditions (Kartsona \& Hilari, 2007). They allow the clinician to understand and measure the impact of disease on the patients' life (Patrick \& Erickson, 1993) and to incorporate the patient's perspective in clinical decision-making (Mayou \& Bryant, 1993). As a result, clinicians can provide better rehabilitation, which can maximize well-being and quality of life (Royal College of Physicians, 2008).

Stroke is a condition that affects many aspects of HRQL (Pickard, Johnson, Feeny, Shuaib, Carriere, \& Nasser, 2004; Northcott \& Hilari, 2011). Several studies have shown that people after stroke experience a decline in their quality of life in terms of depression, physical disabilities, and reduced social support (Carod-Artal, Egido, González, \& Varela de Seijas, 2000). Stroke is the most common cause of aphasia an acquired language disorder that can affect all language modalities: understanding what other people say, expressing oneself, and reading and writing (Chapey, 2008). It is a long-term and lifechanging condition. Aphasia has a profound impact on stroke survivors' HRQL (Hilari, 2011). A recent systematic review reported that emotional distress, reduced level of activities, increased co-morbidity and severity of communication impairment were predictors of poorer HRQL in people with aphasia (Hilari, Needle, \& Harrison, 2012).

Measures used to evaluate HRQL after stroke should be accessible to people with aphasia. The most extensively tested with people with aphasia stroke-specific HRQL scale is the Stroke and Aphasia Quality of Life scale-39 (Hilari, Byng, Lamping, \& Smith, 2003). The SAQOL-39 is a self-report scale carried out in an interview format to facilitate people with aphasia. It covers participants' perception of how stroke and aphasia have affected his/her functioning in four domains: physical, psychosocial, communication and energy. The scale consists of 39 items each scored on a 5-point scale, with high scores indicating better HRQL. The SAQOL-39 has also been evaluated in a generic stroke population, including both people with and without aphasia, producing the SAQOL-39 generic version (SAQOL-39g). The SAQOL-39g has exactly the same items as the SAQOL-39 and it is administered and scored in the same way, but its items group in three (physical, psychosocial and communication) rather than four domains (Hilari, Lamping, Smith, Northcott, Lamb, \& Marshall, 2009). 
The SAQOL-39g can be used with people with any severity of expressive language impairment and moderate - mild recaptive language impairment (Hilari et al., 2003; Hilari et al., 2009). Yet, there still are some people with aphasia, those with severe receptive aphasia who are unable to complete HRQL scales (Hilari \& Byng, 2009). As a result they may be excluded from stroke-outcomes research, clinical outcome data or service evaluation (Cruice, Worrall, Hickson, \& Murison, 2005). To prevent this, proxy responders can be used: significant others of people with aphasia who complete a HRQL scale as they think their partner with aphasia would, if they were able to do it themselves.

If we are to use proxy respondents to report on the HRQL of people with such severe aphasia that they cannot report for themselves, then we need to know how accurate proxies are in their ratings. Literature on proxy-self-report agreement in different clinical populations suggests that a) proxy-self report agreement is better in the longer term because proxies have been longer exposed to their partners' symptoms (Pickard et al., 2004; Sneeuw, Sprangers, \& Aaronson, 2002) and b) proxies tend to report poorer HRQL than the patients report themselves, but agreement is better for more observable domains (Sneeuw et al., 2002). Looking at proxy-self-report agreement on HRQL for people with aphasia, Cruice and colleagues (2005), used generic HRQL scales and found significant differences. Still, in line with the generic proxy literature, there was higher agreement for objective domains (e.g., physical) than subjective domains (e.g., psychological). Hilari, Owen \& Farrelly (2007), used the SAQOL-39 to address the same question. Although there were significant differences between people with aphasia and proxy responses, these differences were small to moderate $(d=0.2-0.5)$ and correlations between proxy and self-report scores were high for the overall score and the physical, psychosocial and communication domain (ICC $=0.7-0.8$ ). These findings are important as they suggest that by using a measure tailored to people with aphasia it is possible for proxy respondents to provide good estimations of the HRQL of their partners with aphasia. However, these findings need to be replicated in different populations.

In terms of factors affecting proxy and self-report agreement on HRQL post stroke, Knapp and Hewison (1999) unlike other studies (Williams, Bakas, Brizendine, Plue, Tu, Hendrie, \& Kroenke, 2006) found no effect for patient or proxy mood, but a significant effect for carer strain, with higher carer strain associated with discrepancy in assessments. Looking specifically at people with aphasia, Hilari et al. found no effect for carer emotional distress or strain (Hilari et al., 2007). The impact of such variables on proxy and self-report agreement on HRQL needs further exploration for people with aphasia.

This study aimed to assess proxy-self-report agreement on the Greek version of the SAQOL-39g (Kartsona \& Hilari, 2007). The Greek SAQOL-39g has strong psychometric properties, with excellent acceptability (minimal missing data; no floor/ceiling effects), test-retest reliability (ICC $=0.96$ scale, 0.83 - 0.99 domains), internal consistency (Cronbach's alpha 0.96 scale, $0.92-0.96$ domains), and convergent $(\mathrm{r}=0.53$ 0.80 scale; $0.54-0.89$ domains) and discriminant validity $(\mathrm{r}=$ 0.52 scale; 0.04 - 0.48 domains) (Efstratiadou, Chelas, Ignatiou, Christaki \& Hilari, 2012). We expected to replicate the findings of previous research. We hypothesized that proxy ratings of the HRQL of people with aphasia will correlate highly with self-report ratings; but proxies will tend to rate people with aphasia as more severely affected than they rate themselves. The possible effect of proxies' high carer strain and emotional distress on level of agreement was also explored.

\section{Methods}

\section{Design and Participants}

An interview-based survey study was carried out. People with aphasia were recruited through Speech and Language Therapy settings in four different cities in Greece and three in Cyprus. They had to meet the following criteria: a) aphasia due to a stroke; b) at least 6 months post stroke and medically stable, c) be able to nominate a significant other to act as a proxy responder and d) score $\geq 7 / 15$ on the receptive subtests of the Frenchay Aphasia Screening Test (FAST) (Enderby, Wood, \& Wade, 1987) which is the cut off score for self-completion of the SAQOL-39g. The inclusion criteria for the proxies were: a) see the person with aphasia at least twice a week; b) older than 18 years old and c) no known severe mental health or cognitive problems.

\section{Procedure and Measures}

The study obtained ethical approval by the City University London School of Health Sciences Research Ethics Committee. Speech and Language Therapy (SLT) in Greece is typically offered in private settings and, therefore, such settings, known to provide services to people were aphasia, were approached in order to find participants. At each site, people with aphasia who met the eligibility criteria were invited to take part along with one significant other to act as a proxy. Interviews took place at the SLT clinic or at the participant's home. All interviews were conducted by a Speech and Language Therapist experienced in working with people with aphasia. First, the receptive subtests of the FAST were completed to ensure participants with aphasia could self-report on the SAQOL-39g. These subtests comprise auditory and reading comprehension. Scores range from 0 - 15 with higher scores suggesting milder aphasia. Then, the person with aphasia completed the SAQOL-39g in an interview format. As indicated above, scores for each domain and the overall scale range from 1 to 5 with higher scores indicating better quality of life. While the person with aphasia was interviewed, proxies completed the following measures by themselves: SAQOL-39g proxy version, General Health Questionnaire-12 item (GHQ-12) (Goldberg, 1972) and the Caregiver Strain Index (CSI) (Robinson, 1983). The GHQ-12 is a measure of emotional distress. Scores on GHQ-12 range $0-12$ and scores $\geq 3$ indicate high emotional distress. The CSI is a 13-question tool that measures strain related to care provision. There is at least one item for each of the following major domains: employment, financial, physical, social and time. Scores on the CSI range $0-13$ and positive responses to seven or more items indicate a greater level of strain.

\section{Data Analysis}

Descriptive statistics were used to summarize data. Proxy and self-report agreement was explored by calculating intraclass correlation coefficients (ICCs). ICC $<0.40$ was seen as indicating poor agreement; 0.40 to 0.75 fair to good agreement, and 0.76 to 1.00 , excellent agreement (Rosner, 2000). Mean responses of the people with aphasia and their proxies were 
compared for each domain and the overall SAQOL-39g with paired-samples t-tests. Effect sizes were calculated by dividing the mean difference scores (proxy minus self-report score) by the standard deviation of the mean difference scores (Marshall, Hays, \& Nicholas, 1994). An effect size of 0.2 was considered a small bias, 0.5 a moderate bias and 0.8 a large bias (Cohen, 1998). Independent sample t-tests were used to compare agreement on the SAQOL-39g (mean difference scores) of those with high versus those with low emotional distress and of those with high versus those with low carer strain.

\section{Results}

\section{Participants}

Twenty three pairs of people with aphasia and their proxies took part in the study. Table 1 details their characteristics. The majority of the people with aphasia were male $19(83 \%$. They ranged in age $39-81$ years, with a mean $(\mathrm{SD})=60.7(11.2)$. Most of them were married/had a partner $20(87 \%)$. Their degrees of receptive aphasia on FAST ranged 7 - 15 [mean (SD) = 11 (2.5)]. Most of the proxies were female $20(87 \%)$. They ranged in age $38-75$ years, with a mean $(\mathrm{SD})=58.1(11.8)$. The majority $19(73 \%)$ were married/partners of the people with aphasia. Proxy scores on the CSI ranged $0-13$ with a mean $(\mathrm{SD})=7.65(4.0)$ and a median of nine. Fifteen $(65.2 \%)$ of them were classified as having high carer strain (CSI scores 7 - 13). Scores on the GHQ-12 ranged $0-9$, with mean (SD) $=$ 4.8 (2.9) and a median of five. Approximately $78 \%$ were classified as having high emotional distress (GHQ-12 scores $\geq 3$ ).

\section{Proxy and Self-Report Agreement}

Table 2 presents participants with aphasia (PWA) and proxies mean scores on the SAQOL-39g and its sub-domains, the difference in mean SAQOL-39g scores (proxy-PWA) and the agreement statistics (ICC, $t$ tests and effect sizes). The proxyPWA difference mean scores were small in all three SAQOL$39 \mathrm{~g}$ domains and in the overall scale $(-0.08)-(-0.35)$. However their standard deviations were comparatively large (0.39 0.75). In all three SAQOL-39g domains and the overall scale, proxies rated PWA as more impaired than PWA rated themselves. There was a significant difference between PWA and proxy reporting for the overall scale $(\mathrm{t}(22)=-2.31, p<0.05)$ and two of its domains, physical $(\mathrm{t}(22)=-2.18, p<0.05)$ and communication $(\mathrm{t}(22)=-2.27, p<0.05)$. However, the biases between PWA and proxies mean scores were small to moderate, with effect sizes ranging from 0.15 to 0.47 .

The strength of the agreement (ICC) between proxies and PWA was excellent for the overall SAQOL-39g scale (0.96) and all three domains: physical (0.97), psychosocial (0.89) and communication (0.79).

Independent sample $t$ tests were used to evaluate the possible effect of proxy carer strain and emotional distress on the agreement. We compared proxy-PWA difference mean scores on the SAQOL-39g of those with high carer strain (CSI score $\geq$ 7) versus those with low, and of those with high emotional distress (GHQ score $\geq 3$ ) versus those with low. The results were not significant.

\section{Discussion}

This study explored the proxy and self-report agreement on
Table 1.

Respondent characteristics: participants with aphasia $(\mathrm{n}=23)$ and their proxies $(n=23)$.

\begin{tabular}{|c|c|c|}
\hline Variable & PWA n (\%) & Proxies n (\%) \\
\hline \multicolumn{3}{|l|}{ Sex } \\
\hline Female & $4(17)$ & $20(76.9)$ \\
\hline Male & $19(83)$ & $3(11.5)$ \\
\hline \multicolumn{3}{|l|}{ Age (y) } \\
\hline Mean (SD) & $60.7(11.2)$ & $58.1(11.8)$ \\
\hline Range & $39-81$ & $38-75$ \\
\hline \multicolumn{3}{|l|}{ Marital status } \\
\hline Married/had partner & $20(87)$ & $21(92)$ \\
\hline Single & $1(4)$ & $1(4)$ \\
\hline Divorced/widowed & $2(9)$ & $1(4)$ \\
\hline \multicolumn{3}{|l|}{ Tim post onset } \\
\hline Mean (SD) & $45.7(35.4)$ & - \\
\hline Range & $9-162$ & - \\
\hline $6 m-2 y$ & $7(30)$ & - \\
\hline $2 y-4 y$ & $8(35)$ & - \\
\hline $4 \mathrm{y}+$ & $8(35)$ & - \\
\hline \multicolumn{3}{|c|}{ Relationship to person with aphasia } \\
\hline Spouse & - & $18(69.2)$ \\
\hline Child & - & $1(3.8)$ \\
\hline Partner & - & $1(3.8)$ \\
\hline Other family & - & $3(11.5)$ \\
\hline \multicolumn{3}{|l|}{ GHQ - 12} \\
\hline Mean (SD) & - & $4.8(2.9)$ \\
\hline Range & - & $0-9$ \\
\hline \multicolumn{3}{|l|}{ CSI } \\
\hline Mean (SD) & - & $7.7(4.0)$ \\
\hline Range & - & $0-13$ \\
\hline
\end{tabular}

FAST: Frenchay Aphasia Screening Test; GHQ-12: General Health Questionnaire-12; CSI: Caregiver Strain Index.

the Greek SAQOL-39g and the possible effect of proxy carer strain and emotional distress on this agreement. We found no effect of proxy level of distress or strain on the level of agreement. Proxy scores were significantly lower than the scores of their partners with aphasia on the overall scale and on two of the three domains: physical and communication. The standard deviations of the difference scores were large, showing that proxy responses may not be a good indicator for self-report responses at the individual level. At the group level however, the results were encouraging. Although proxies rated their partners HRQL consistently lower, the effect sizes were small to medium $(0.15-0.47)$ and they were comparable to those of other stroke specific HRQL scales, i.e. the Stroke Impact Scale (SIS) $(0.1$ - 0.4) (Duncan, Lai, Tyler, Perera, Reker, \& Studenski, 2002). Considering that people with aphasia were excluded from that study and yet comprised the sample in this study, these results are very positive. They also compare favourably to Cruice et al.'s findings with people with aphasia and their 
Table 2

Comparison of participants' scores on the stroke and aphasia quality of life scale-39g (SAQOL-39g) $($ pairs, $n=23)$.

\begin{tabular}{|c|c|c|c|c|c|c|}
\hline SAQOL-39g & PWA mean (SD) & Proxies mean (SD) & Proxy-PWA difference mean (SD) & $\mathrm{ICC}$ & $\mathrm{t}$ & Effect size \\
\hline Physical & $3.38(1.20)$ & $3.20(1.22)$ & $-0.18(0.39)$ & 0.97 & $-2.18^{*}$ & 0.46 \\
\hline Psychosocial & $3.28(0.83)$ & $3.20(0.88)$ & $-0.08(0.54)$ & 0.89 & -0.69 & 0.15 \\
\hline Communication & $3.53(0.83)$ & $3.18(0.97)$ & $-0.35(0.75)$ & 0.79 & $-2.27^{*}$ & 0.46 \\
\hline Overall scale & $3.36(0.77)$ & $3.20(0.90)$ & $-0.16(0.34)$ & 0.96 & $-2.31^{*}$ & 0.47 \\
\hline
\end{tabular}

PWA: participants with aphasia; ICC: intra-class correlation coefficient; ${ }^{*} p<0.05$.

proxies where effect sizes with the SF-36 (4/8 scores $\geq 0.6$ and up to 1.24$)$ and the Dartmouth COOP Charts (4/9 scores $\geq 0.25$ and up to 0.84) were larger (Cruice et al., 2005).

Moreover, proxy and self-report agreement on the Greek SAQOL-39g as measured by ICCs was excellent $(0.79-0.97)$. These results are promising, given that studies with small sample sizes ( $\mathrm{n}<50$ pairs) tend to report lower levels of agreement (Sneeuw et al., 2002). The high agreement in this study could be attributed to the fact that the SAQOL-39g is a measure specifically adapted for people with aphasia and thus potentially more relevant and accessible to them than the scales used in other studies. It could also be related to the fact that all proxy raters were relatives of the people with aphasia rather than paid carers or health care providers. Level of agreement between patients and health care providers is lower than that between patients and significant others (Sneeuw et al., 2002). Although it makes sense to predict that health care providers may be less aware of patients' views on their HRQL than family members, this is not always the case in other clinical groups (Grassi, Indelli, Maltoni, Falcini, Fabbri, \& Indelli, 1996; Sneeuw, Aaronson, Sprangers, Detmar, Wever, \& Schornagel, 1999). Thus, it is useful to confirm this effect for people with aphasia. High levels of agreement may be also related to time post-onset. Our sample comprised people with aphasia who were six months post-stroke or more. Evidence in the literature suggests that agreement tends to be higher in long term patients (Pickard et al., 2004) as their proxies have had longer exposure to their symptoms.

Of particular note is the high agreement between self-report and proxy ratings on the Greek SAQOL-39g psychosocial domain (ICC $=0.89$, difference not significant). Sneeuw et al.'s systematic review (2002) suggests that median correlations of proxy-self-report agreement on psychosocial domains tend to be moderate $(0.48-0.50)$. In stroke studies, reported correlations are: for the psychosocial domain of the Sickness Impact Profile 0.61 (Sneeuw, Aaronson, de Haan \& Limburg, 1997), for emotional functioning on the EuroQol 0.30 (Dorman, Waddell, Slattery, Dennis, \& Sandercock, 1997), for anxiety and depression on the EQ-5D 0.43 (Pickard et al., 2004), for all Stroke-Specific Quality of Life scale (SS-QOL) domains 0.30 0.59 (Williams et al., 2006) and for the memory, emotion, communication and social participation domains of the SIS 0.50 - 0.56 (Duncan et al., 2002).

Looking at the level of agreement from a different perspective we noted that proxy and self-report agreement for the overall scale $(0.96)$ was the same as its test-retest reliability $(0.96)$ (Efstratiadou et al., 2012). This means that for our sample, the strength of proxy and patient agreement was the same as the test-retest reliability of the patient on the Greek SAQOL-39g. As far as the domains are concerned, for the communication domain scores were different $(0.79$ for proxy-self-report agreement and 0.90 for test-retest reliability). However, ICCs on the proxy and self-report agreement for the physical (0.97) and the psychosocial domain $(0.89)$ were very similar to those for test-retest reliability ( 0.99 and 0.83 respectively) (Efstratiadou et al., in press). These comparisons overall suggest a good level of proxy-self-report agreement on HRQL using the Greek SAQOL-39g. Patients' self-reports are often taken as a gold standard. However, Sneeuw and colleagues suggest that, like proxy ratings, patient ratings are not perfectly reliable and they could be also subject to several forms of bias (Sneeuw et al., 2002).

In our sample, the level of agreement was not associated with carer strain and emotional distress. These findings are in line with previous research with the SAQOL-39 with people with aphasia (Hilari et al., 2007) and, in terms of the effect of carer mood, with other stroke studies (Knapp \& Hewison, 1999). However, unlike other stroke studies (Williams et al., 2006; Knapp \& Hewison, 1999) we found no effect for carer strain. This may be due to the overall good levels of agreement in our sample and also to the fact that our sample were all longer term post stroke.

Strengths of the study included the use of an accessible and relevant to people with aphasia measure, the Greek SAQOL$39 \mathrm{~g}$, the use of face to face interviews and the facilitation of the participants with aphasia by a Speech and Language Therapist. All this ensured that all people with aphasia who took part in the study were able to self-report on all the measures used and there were no missing data.

A limitation of this study was the small sample size. A larger sample size would have allowed us to explore in more depth the potential factors that could affect proxy-self-report agreement. For example, there was not enough variability in our data to explore potential effects of demographic variables. Yet in other studies the level of agreement was not related to demographics variables (Choiniere, Melzack, Girard, Rondeau, \& Paquin, 1990; McCusker \& Stoddard, 1984; O’Brien \& Francis, 1988). Other factors that could have affected agreement, and could be explored in future research, include participant with aphasia's mood, cognitive status and aphasia severity. Considerations of respondent burden prevented us from exploring their mood and cognition. Whether levels of agreement are similar or different in people with severe aphasia is an important issue. However, people with very severe receptive aphasia would not be able to take part in such research as they would not be able to selfreport on the measures used (Hilari \& Byng, 2009). Moreover, in their review, Sneeuw et al. (2002) found that the relationship between agreement and severity was U-shaped (i.e., agreement was better for very good or very poor health status and worse for moderately impaired health status). Larger studies could 
also explore factors such as time spent with the person with aphasia, education and carer levels of social support. Lastly, future research could explore proxy and self-report agreement on the Greek SAQOL-39g in a generic stroke sample including those without aphasia.

\section{Conclusion}

Using the Greek SAQOL-39g, proxy raters can provide useful information on the HRQL of people with severe aphasia. However, the client is the most appropriate source of information on their own HRQL and proxy reports should be used only when people with aphasia are so severely affected that they are unable to self-report. In addition, clinicians and researchers need to be aware of trends in proxy reporting and take these into account when interpreting data.

\section{REFERENCES}

Berzon, R., Hays, R. D., \& Shumaker, S. A. (1993). International use, application and performance of health-related quality of life instruments. Quality of Life Research, 2, 367-368. doi:10.1007/BF00422214

Bullinger, M., Anderson, R., Cella, D., \& Aaronson, N. K. (1993). Developing and evaluating cross cultural instruments: From minimum requirements to optimal models. Quality of Life Research, 2, 451-459. doi:10.1007/BF00422219

Carod-Artal, J., Egido, J. A., González, J. L., Varela de Seijas, E. (2000). Quality of life among stroke survivors evaluated 1 year after stroke: Experience of a stroke unit. Stroke, 31, 2995-3000. doi:10.1161/01.STR.31.12.2995

Chapey, R. (2008). Language intervention strategies in aphasia and related neurogenic communication disorders (5th ed.). Baltimore: Lippincott Williams \& Wilkins.

Choiniere, M., Melzack, R., Girard, N., Rondeau, J., \& Paquin, M. J. (1990). Comparisons between patients' and nurses' assessment of pain and medication efficacy in severe burn injuries. Pain, 40, 143152. doi:10.1016/0304-3959(90)90065-L

Cohen, J. (1988). Statistical power analysis for the behavioural sciences (2nd ed.). Hillsdale, NY: Lawrence Erlbaum.

Cruice, M., Worrall, L., Hickson, L., \& Murison, R. (2005). Measuring quality of life: Comparing family members' and friends' ratings with those of their aphasic partners. Aphasiology, 19, 111-129. doi:10.1080/02687030444000651

Dorman, P. J., Waddell, F., Slattery, J., Dennis, M., \& Sandercock, P. (1997). Are proxy assessments of health status after stroke with the EuroQol questionnaire feasible, accurate, and unbiased? Stroke, 28, 1883-1887. doi:10.1161/01.STR.28.10.1883

Duncan, P. W., Lai, A. M., Tyler, D., Perera, S., Reker, D. M., \& Studenski, S. (2002). Evaluation of proxy responses to the stroke impact scale. Stroke, 33, 2593-2599. doi:10.1161/01.STR.0000034395.06874.3E

Efstratiadou, E. A., Chelas, E. N., Ignatiou, M., Christaki, V., Papathanasiou I., \& Hilari, K. (2012) Quality of life after stroke: Evaluation of the Greek SAQOL-39g. Folia Phoniatrica Logopaedica, 64 doi: $10.1159 / 000340014$

Enderby P., Wood V., \& Wade, D. (1987). Frenchay aphasia screening test. Windsor: NFER-Nelson.

Goldberg, D. P. (1972). The detection of psychiatric illness by questionnaire. London: Oxford University Press.

Grassi, L., Indelli, M., Maltoni, M., Falcini, F., Fabbri, L., \& Indelli, R. (1996). Quality of life of homebound patients with advanced cancer: Assessments by patients, family members, and oncologists. Journal of Psychosocial Oncology, 14, 31-45. doi:10.1300/J077v14n03 03

Hays, R. D., Anderson, R., \& Revicki, D. (1993). Psychometric considerations in evaluating health-related quality of life measures. Quality of Life Research, 2, 441-449. doi:10.1007/BF00422218

Hilari, K. (2011). The impact of stroke: Are people with aphasia dif- ferent to those without? Disability and Rehabilitation, 33, 211-218. doi:10.3109/09638288.2010.508829

Hilari, K., \& Byng S. (2009). Health-related quality of life in people with severe aphasia. International Journal of Language and Communication Disorders, 44, 193-205. doi: $10.1080 / 13682820802008820$

Hilari, K., Byng, S., Lamping, D. L., \& Smith, S. C. (2003). Stroke and aphasia quality of life scale-39 (SAQOL-39): Evaluation of acceptability, reliability and validity. Stroke, 34, 1944-1950. doi:10.1161/01.STR.0000081987.46660.ED

Hilari, K., Lamping, D. L., Smith, S. C., Northcott, S., Lamb, A., \& Marshall, J. (2009). Psychometric properties of the stroke and aphasia quality of life scale (SAQOL-39) in a generic stroke population. Clinical Rehabilitation, 23, 544-557. doi: $10.1177 / 0269215508101729$

Hilari, K., Needle, J. \& Harrison, K. (2012). What are the important factors in health-related quality of life for people with aphasia? A systematic review. Archives of Physical Medicine and Rehabilitation, 93, S86-S95. doi:10.1016/j.apmr.2011.05.028

Hilari, K., Owen, S., \& Farrelly, S. J. (2007). Proxy and self-report agreement on the stroke and aphasia quality of life scale-39. Journal of Neurology, Neurosurgery and Psychiatry, 78, 1072-1075. doi:10.1136/jnnp.2006.111476

Kartsona, A., \& Hilari, K. (2007). Quality of life in aphasia: Greek adaptation of the stroke and aphasia quality of life scale-39 item (SAQOL-39). Europa Medicophysica, 43, 27-35.

Knapp, P., \& Hewison, J. (1999). Disagreement in patient and carer assessment of functional abilities after stroke. Stroke, 30, 934-938. doi:10.1161/01.STR.30.5.934

Marshall, G. N., Hays, R. D., \& Nicholas, R. (1994). Evaluating agreement between clinical assessment methods. International Journal of Methods in Psychiatric Research, 4, 249-257.

Mayou, R., \& Bryant, B. (1993). Quality of life in cardiovascular disease. British Medical Journal, 69, 460-466.

McCusker, J., \& Stoddard, A. M. (1984). Use of a surrogate for the sickness impact profile. Medical Care, 22, 789-795. doi:10.1097/00005650-198409000-00002

Northcott, S., \& Hilari K. (2011). Why do people lose their friends after a stroke? International Journal of Language and Communication Disorders, 46, 524-534doi:10.1111/j.1460-6984.2011.00079.x

O'Brien, J., \& Francis, A. (1988). The use of next-of-kin to estimate pain in cancer patients. Pain, 35, 171-178. doi:10.1016/0304-3959(88)90224-2

Patrick, D. L., \& Erickson, P. (1993). Assessing health-related quality of life for clinical decision making. In the S. R. Walker (Eds.), Quality of life assessment: Key issues in the 1990's (pp. 11-63). Dordrecht: Kluwer Academic Publishers.

Pickard, A. S., Johnson, J. A., Feeny, D. H., Shuaib, A., Carriere, K. C., \& Nasser, A. M. (2004). Agreement between patient and proxy assessments of health-related quality of life after stroke using the EQ5D and health utilities index. Stroke, 35, 607-612. doi:10.1161/01.STR.0000110984.91157.BD

Robinson, B. (1983). Validation of a caregiver strain index. Journal of Gerontology, 38, 344-348.

Rosner, B. (2000). Fundamentals of biostatistics (6th ed.). Pacific Grove, CA: Duxbury.

Royal College of Physicians (2008). National clinical guidelines for stroke (3rd ed.). Prepared by the Intercollegiate Working Party for Stroke. London: RCP.

Sneeuw, K. C. A., Aaronson, N. K., de Haan, R. J., \& Limburg, M. (1997). Assessing quality of life after stroke. The value and limitations of proxy ratings. Stroke, 28, 1541-249. doi:10.1161/01.STR.28.8.1541

Sneeuw, K. C. A., Sprangers, M. A., \& Aaronson, N. K. (2002). The role of health care providers and significant others in evaluating the quality of life of patients with chronic disease. Journal of Clinical Epidemiology, 55, 1130-1143. doi:10.1016/S0895-4356(02)00479-1

Williams, L. S., Bakas, T., Brizendine, E., Plue, L., Tu, W., Hendrie, H., \& Kroenke, K. (2006). How valid are family proxy assessments of stroke patients' health-related quality of life? Stroke, 37, 2081-2085. doi:10.1161/01.STR.0000230583.10311.9f 